\title{
Benefits of Oral Contraceptive Pill Pretreatment in Endometriosis for IVF/ICSI-ET: A Retrospective Cohort Study
}

\author{
Sangita Chakrabartty ${ }^{1,2}$, ${ }^{*}$ Hanwang Zhang ${ }^{1,2}$, Xiyuan Dong ${ }^{1,2}$ and Martin Omondi Alfred ${ }^{3}$ \\ ${ }^{1}$ Reproductive Medicine Center, Tongji Hospital, Wuhan, PR China \\ ${ }^{2}$ Tongji Medical College, Huazhong University of Science and Technology, Wuhan, PR China \\ ${ }^{3}$ China Pharmaceutical University, Department of Pharmacology, Nanjing, PR China
}

\begin{abstract}
Pretreatment with oral contraceptive pills (OCP) has been widely applied in IVF/ICSI -ET treatment. However, it remains unclear whether OCP influences pregnancy rate of fresh cycle and subsequent cumulative pregnancy rate in patients with endometriosis. In this retrospective study, a total of 512 first attempt IVF/ICSI cycles of patients with endometriosis were included and divided into two groups: the study group included 216 cycles of patients undergoing pretreatment with OCP before pituitary suppression and ovarian stimulation, while 296 cycles of patients without pretreatment were considered as control. The ovarian stimulation performance and pregnancy results were compared between the two groups. Patients with OCP pretreatment required shorter duration, a lower dosage of gonadotropins, but obtained higher E2 level and more retrieved oocytes. Patients in the two groups obtained comparable fertilization rate, cleavage rate, but the number and rate of day 3 high-quality embryos were higher in OCP-pretreated patients. No differences were found in IVF/ICSI-ET outcomes, in terms of implantation rate, clinical pregnancy rates, live birth pregnancy rate, and cumulative pregnancy rate. In addition, despite the lack of statistical significance, a trend toward higher clinical pregnancy rate in a fresh cycle and cumulative pregnancy rate was found in OCP pretreated patients. It should be recommended that patients with endometriosis take OCP pretreatment before ovarian stimulation, since it offers advantages of lower cost and higher embryo-quality, and does not negatively influence pregnancy rates.
\end{abstract}

Key Words: Endometriosis, oral contraceptive pills, IVF/ICSI-ET, pregnancy.

\section{INTRODUCTION}

Endometriosis is a common condition in women of reproductive age. In addition to pain, endometriosis may also reduce fertility. The causes of infertility in women with endometriosis may range from anatomical distortions due to adhesions and fibrosis to endocrine abnormalities and immunological disturbances (Tanbo and Fedorcsak, 2017). Several studies have revealed that endometriosis lesions are associated with hormonal imbalance such as estrogen synthesis, metabolism and responsiveness and progesterone resistance. The hormonal changes affect the ability of endometrial cells to migrate, proliferate and to infiltrate the mesothelium, leading to infertility, inflammation and pain. Hormonal imbalance in endometriosis represents a viable target for treatment (Pluchino et al., 2016).

Estrogen and progesterone effects on these inflammatory processes are tightly and reciprocally regulated through the expression of steroid receptors, cofactors, chaperone proteins, and downstream signaling components. In endometriosis, the inflammatory cascades, normally witnessed during menstruation is normally activated prematurely and endogenous endometrial mechanisms of inflammation resolution appear as being defective. The interim abnormal inflammation is also related to an imbalance between estrogen and progesterone actions; the normal luteal-phase dominance of progesterone action appears to be lost and is replaced by

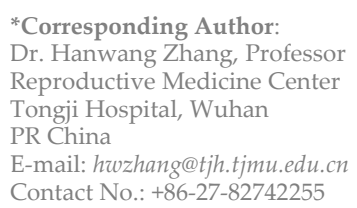

progesterone resistance and estrogen dominance (Lessey and Young, 2014).

Endometriosis appears to be an estrogen-dependent condition. Suppression of ovarian activity may result in the inactivation of endometriosis. Oral contraceptive pills (OCP) has been extensively used to suppress ovarian activity and this has resulted to this agent has become a standard treatment for pain and infertility associated with endometriosis. Hormonal dependency has elicited common use of agents which suppress ovarian activity. In the 1970's, Danazol was identified as such an agent and quickly became a standard treatment of pain and sub-fertility associated with endometriosis. Other agents such as progestins and/or oral contraceptives have also been used (Hughes et al., 2003).

Medical treatments of endometriosis include combined oral contraceptive pills, progestogens, androgen hormone and gonadotrophin-releasing hormone analogs $(\mathrm{GnRH} \alpha)$ (Vercellini et al., 2016). Combined OCP consists of estrogen and progestogen, which inhibit the women's own production of follicle stimulating hormone (FSH) and Luteinizing hormone ( $\mathrm{LH})$ through a negative feedback. The combined OCPs suppress gonadal function, and ovulation can be prevented in the absence of an LH-surge. Synthetic Progestogen preparations prevent the occurrence of a spontaneous LH surge during ovarian stimulation in patients whose endogenous gonadotropins were GnRH agonists (Levens et al., 2009).

The estrogen component will avoid FSH rise and follicle recruitment and progestogen will avoid leutinizing hormone rise. Recently, OCPs were also shown to downregulate cell proliferation and increase apoptosis in the eutopic endometrium of women with endometriosis (Davis et al., 2007). 
With different suggested options, oral contraceptive, in particular, is one of the most favorable options for clinicians for cycle planning in IVF/ICSI. Controversy has however arisen over the last few years, as contradictory data have been published on whether the use of OCPs in the previous cycle might have a deleterious effect on subsequent IVF/ICSI cycle outcome. However, no differences have been found in pregnancy rate, whether or not they receive OCP (Bellver et al., 2007).

Gonadotropin releasing hormone agonist $(\mathrm{GnRH} \alpha)$ is used to constant stimulation of pituitary. During in vitro fertilization-embryo transfer (IVF-ET) cycles, most women undergo hypothalamic pituitary suppression prior to the initiation of the ovarian stimulation. This allows better timeline of cycles, synchronization of follicular development, and prevents untimely ovulation. The gonadotropin quelling is usually achieved by the use of gonadotropin releasing hormone agonist (Ma et al., 2007; Dessolle et al., 2011).

Hormonal deregulation with a gonadotropin releasing hormone (GnRH) agonist has been used in the field of assisted reproductive technology (ART). It causes repression of a premature luteinizing hormone (LH) surge, improvement of follicular recruitment, development, and confirmation of follicular and endometrial development (Siristatidis et al., 2015). It assists in planning the treatment and provides the convenience of both the fertility workload and the patients. The limitation of deregulation has increased the cost of treatment (Venetis et al., 2009). Since the introduction of gonadotrophins (Gn) into controlled ovarian hyper stimulation $(\mathrm{COH})$, it has played a leading role in the IVF/ICSI procedure (Zhu et al., 2016). GnRH agonists $(\mathrm{GnRH}-\mathrm{a})$ produce an initial hypersecretion of pituitaryderived gonadotropins followed by sustained pituitary desensitization and cessation of gonadotropin function by yet inadequately understood mechanisms. These properties have been exploited by altering the dosage and the timing of administration during stimulation to produce contrasting results among various target populations. The use of GnRH- $\alpha$ in assisted reproductive technology (ART) has been to prevent premature luteinization, to reduce cycle cancellation, and to improve oocyte recovery rates (Levens et al., 2009).

The aim of this study was to compare the response to stimulation, and IVF cycle outcomes utilizing GnRHa and pretreated with OCPs and without OCPs on patients who had visited the Tongji University Hospital.

\section{PATIENTS AND METHODS}

\section{Study location}

This is a retrospective study and was conducted in the Department of Reproductive center of Tongji Hospital, Tongji Medical College, Huazhong University of Science and Technology (HUST), Wuhan, China.

\section{Data Collection}

We collected the data from electronic record system in our institution. It includes individual patient's demographic and clinical information with treatment records, pregnancy outcome, and obstetric records.

\section{Inclusion Criteria}

All the patients diagnosed with endometriosis by diagnostic or therapeutic methods.

We included mild and moderate endometriosis cases in our study.
Mild (Stage II) endometriosis

Findings restricted to only superficial lesions and possibly a few filmy adhesions, some deep lesions are present in the cul-de-sac.

\section{Moderate (Stage III)}

Findings restricted to only superficial lesions and possibly a few filmy adhesions plus the presence of endometriomas on the ovary and more adhesions.

\section{Study Population}

Between January 2011 to December 2012, GnRH agonist protocol was used in 512 patients with the first attempt. Patients with endometriosis were included and divided into two groups in which 216 patients undergoing pretreatment with OCP before pituitary suppression and ovarian stimulation, while 296 cycles of patients without pretreatment were considered as control. OCP pretreatment was administered for beneficial effect in cycle outcome and cycle schedule. 512 patients met the inclusion criteria for subsequent analysis. The approval of the Institution Review Broad (IRB) was obtained from the Tongji Hospital before the initiation of the study. All patients gave written informed consent for their clinical records to be used in this study. Patient's information was anonymized prior to the analysis.

\section{Methods}

We studied the incidence, hormonal profile, ovulation induction protocols, treatment modalities and outcome in patients with endometriosis with or without OCP associated with infertility group. The decision whether or not to use the treatment is entirely the decision of the couple. The endpoint of the treatment was an ongoing pregnancy beyond 12 weeks of gestation, that is, the end of the first trimester. Once the diagnosis was made, we have assured the patient's eligibility and treatment modalities for the down-regulation and controlled ovarian hyper stimulation $(\mathrm{COH})$ followed by IVF/ICSI-ET.

\section{Treatment protocol for ovarian hyper stimulation}

Good controlled ovarian hyper stimulation leads to multiple synchronized developed follicles and high quality of mature oocytes, thus improves fertilization rate and pregnancy rate. We emphasized on long protocol with Gonadotrophin releasing hormone $(\mathrm{GnRH})$ agonist for down-regulation followed by Gonadotrophin (rFSH, $\mathrm{HMG}$ ) for induction of ovulation. In patients without OCP pretreatment, subcutaneous injection of $0.1 \mathrm{mg} \mathrm{GnRH}$ (Decapeptyl, Ferring, Switzerland) or (Dipherelin, Ipsen, Australia) was initiated daily from the mid-luteal phase of the preceding cycle, which was reduced to $0.5 \mathrm{mg}$ once adequate down-regulation was achieved. As for patients with OCP pretreatment, administration of OCP (Marvelon, Organon, Netherlands) was started from the day 3-5 of the previous cycle and commenced for consecutive 21 days. Downregulation was started with $0.1 \mathrm{mg}$ per day.

The final maturation was induced, ovulation was triggered with $250 \mathrm{mcg}$ by an injection of Human chorionic gonadotropin (HCG) alfa (ovitrelle, Serono, Switzerland), which was administered as soon as two leading follicles reached $>17 \mathrm{~mm}$ mean diameter size and endometrial thickness of $7 \mathrm{~mm}$ by ultrasound scan. After 3 days (72 hours) of oocyte retrieval, if endometrial thickness was adequate (at least $>7 \mathrm{~mm}$ ), embryo transfer was performed. A maximum of 2 embryos was transferred except in unusual circumstances. Chemical pregnancy was detected by serum Beta-human chorionic gonadotropin $(\beta \mathrm{HCG})$ analysis 
on day 12 of embryo transfer and was repeated again on day 16 to see doubling time. The transvaginal sonographic scan was scheduled 2 weeks after ET to detect the gestational sac into the uterine cavity to confirm the intrauterine pregnancy and excluded ectopic pregnancy.

Each pregnant woman was followed by ultrasound scan until fetal heart sound was documented (clinical pregnancy). The patient was then referred to an obstetrician for further follow-up. Therefore, the main outcome of the study was clinical pregnancy and live birth of a healthy baby.

According to the Chinese law (Ministry of health) and International health protocol, we routinely replaced two embryos. Conventional IVF was performed in 169 couples in OCP groups and 226 couples in the non-OCP group and 70 patients in non-OCP group and 47 couples in OCP group were performed in ICSI.

\section{Statistical Analysis}

The variables of interest in this study were analyzed using SPSS. Factors being treatment with OCP and non-OCP. Procedures for proportions (fertilization rate, implantation rate etc.) involved a transformation onto the logistic scale, after which maximum likelihood was used to carry out the analysis. For ease of interpretation, however, the results from those analyses were presented on the original scale of proportion. A p-value of $<0.05$ was considered to be statistically significant. All analysis was performed with the SPSS19.0 package (SPSS, 19.0 software Inc., Chicago, IL, USA).

\section{RESULTS}

A total of 512 patients were identified in the electronic records for analysis. The demographic and clinical characteristics of the patients are listed in Table 1. The patients in the non-OCP group were older $(31.4 \pm 3.8$ versus $30.1 \pm 3.3, \mathrm{p}<0.01)$, had a lower AFC (10.1 5.7 versus $13.8 \pm 5.4, \mathrm{p}<0.01)$ and had a higher baseline serum FSH level $(7.9 \pm 4.7$ versus $7.1 \pm 2.0, \mathrm{p}<0.03)$ than the patients in $\mathrm{OCP}$ group. No differences in the BMI, duration of infertility, infertility type, the day 3 endometrial thickness or baseline serum levels of LH and E2 were found between the groups. The antral follicle count (AFC) in both ovaries taken together.

Due to the difference between the two pretreatment protocols, all parameters of ovarian stimulation were comparable between the groups (Table 2). Duration of stimulation with gonadotropin is significantly more days in non-OCP compared to OCP groups $(11.1 \pm 2.4$ versus $10.6 \pm 2.2, p=0.02$ ) and even dosages of gonadotropin are required more in the non-OCP group compared to OCP group (3042.4 \pm 1136.7 versus $2441.3 \pm 938.7, \mathrm{p}<0.01)$. more oocyte retrieval in OCP group than non-OCP group (12.5 \pm 6.5 versus $9.7 \pm 6.7, p<0.01$ ) higher rate and number of day 3 high quality embryos in OCP group $(5.6 \pm 4.1$ versus $4.2 \pm$ $2, \mathrm{p}<0.01$ ) and rate of day 3 high-quality embryos significantly higher in OCP group compared with non-OCP group $(74.7(1219 / 1632)$ versus $71.0(1243 / 1751, p=0.02)$. No significant differences were observed in endometrial thickness on the day of hCG, fertilization method, fertilization rate and cleavage rate between groups.

The final IVF/ICSI-ET outcomes were analyzed in both groups (Table 3). Stimulation was canceled in $57 \mathrm{cy}-$ cles in non-OCP group and 41 in OCP group due to low response and few patients did not start an IVF cycle after the initial consultation for personal reasons.
The biochemical pregnancy rate was 4 out of 239 in non-OCP groups and 10 out of 175 in OCP group. The miscarriage was 28 in 149 and 18 in 112 in OCP group and ectopic pregnancy rate in non-OCP was 1 and 3 in OCP group. Other outcomes like implantation rate, clinical pregnancy rate, live birth rate, miscarriage and ectopic pregnancy rate were statistically not significant.

\section{DISCUSSION}

The present study evaluated the effectiveness of oral contraceptive pill pretreatment with GnRH agonist in endometriosis patients undergoing IVF/ICSI shown in Table 1. Total dose and duration of gonadotropins used for OCP were significantly lower than the non-OCP group, which suggests the advantage of OCP with GnRH agonist protocol. In patients with endometriosis elevated basal serum FSH levels by diminished ovarian reserve, antral follicle sizes during the early follicular phase are often markedly heterogeneous because FSH-sensitive follicles are early exposed to gradient FSH concentrations during the preceding luteal phase (Table 2). Therefore, in endometriosis patients, ovarian stimulation without pituitary suppression is likely to induce asynchronous follicular development and a limited number of dominant follicles. The COS results of the present study may result from pituitary suppression prior to ovarian stimulation.

The numbers of mature oocytes retrieved, day 3 highquality embryos rate and a number of day 3 high-quality embryos were significantly higher in $\mathrm{OCP}$ group as shown in table 3. This implicates OCP pretreatment with $\mathrm{GnRH}$ agonist is effective in improving the ovarian response to COS and IVF/ICSI-ET outcome in patients with endometriosis. Although the length of the OCP exposure varied considerably within each pretreatment group and between the 2 groups and we strongly maintained the 5 days' washout period in each pretreatment group and we observed a considerable difference in $\mathrm{OCP}$ and non-OCP group.

Furthermore, the weekend oocyte retrieval can be significantly reduced by using OCPs which is well known, using this method, therefore, means there is no need to prolong the duration of GnRH agonist treatment (ZegersHochschild et al., 2009). Despite these advances, the pregnancy and miscarriage rates were similar between the 2 groups (table 3). Pregnancy outcomes were similar in the 2 groups but significantly fewer patients were canceled due to poor response may be due to premature LH surge in OCP group (Bendikson et al., 2006). In terms of clinical pregnancy rate per initiated cycle or per embryo transfer cycle in our study, OCP pretreatment with GnRH seemed to work better than without OCP pretreatment but the difference was not statistically significant. OCP pretreatment with $\mathrm{GnRH}$ agonist protocol is considered as beneficial regimens in endometriosis patients as shown in table 2.

The OCP pretreatment in IVF/ICSI cycles is the simplest method for cycle scheduling, and it can be beneficial in improving ovarian responses by inhibition of intrinsic gonadotropins before ovarian stimulation (Biljan et al., 2000). Our result shows no significant changes in pregnancy rates and cycle cancellation rate was also not significant (57/296 in the non-OCP group and 41/216 in OCP group) (Table 3 ). The OCP pretreatment resulted in an increase in the number of mature follicles and in the number of oocyte retrieval. Finally, in our study, the implantation rate and pregnancy rates were not affected by the use of OCP pretreatment. The present study demonstrated that GnRH agonist with OCP pretreatment is 
Table 1: Demographics and clinical characteristics.

\begin{tabular}{|c|c|c|c|}
\hline & Non-OCP group & OCP group & $P$ value \\
\hline No. of cycles & 296 & 216 & \\
\hline Mean age (years) & $31.4 \pm 3.8$ & $30.1 \pm 3.3$ & $<0.01$ \\
\hline $\operatorname{BMI}\left(\mathrm{kg} / \mathrm{m}^{2}\right)$ & $20.8 \pm 2.3$ & $21.1 \pm 2.6$ & NS \\
\hline Duration of infertility (years) & $5(5-20)$ & $5(1-18)$ & NS \\
\hline \multicolumn{4}{|l|}{ Infertility type } \\
\hline Primary infertility $(\%)$ & $61.1(181 / 296)$ & $60.6(131 / 216)$ & NS \\
\hline Secondary infertility (\%) & $38.9(115 / 296)$ & $39.4(85 / 216)$ & NS \\
\hline AFC & $10.1 \pm 5.7$ & $13.8 \pm 5.4$ & $<0.01$ \\
\hline Day 3 endometrial thickness (mm) & $5.3 \pm 1.9$ & $5.2 \pm 1.8$ & NS \\
\hline Baseline serum FSH level (mIU) & $7.9 \pm 4.7$ & $7.1 \pm 2.0$ & 0.03 \\
\hline Baseline serum LH level (mIU) & $4.4 \pm 2.2$ & $4.7 \pm 2.4$ & NS \\
\hline Baseline serum E2 level (pg/mL) & $61.6 \pm 28.2$ & $60.8 \pm 40.0$ & NS \\
\hline
\end{tabular}

Note: $B M I=$ Basic Metabolic rate, AFC = Antral Follicle Count, NS = not statistically significant

Table 2: Controlled Ovarian Hyper stimulation laboratory parameters.

\begin{tabular}{|c|c|c|c|}
\hline & Non-OCP group & OCP group & $P$ value \\
\hline No. of cycles & 296 & 216 & \\
\hline Duration of gonadotropins (days) & $11.1 \pm 2.4$ & $10.6 \pm 2.2$ & 0.02 \\
\hline Dosage of gonadotropins (IU) & $3042.4 \pm 1136.7$ & $2441.3 \pm 938.7$ & $<0.01$ \\
\hline Endometrial thickness on the day of hCG $(\mathrm{mm})$ & $11.4 \pm 2.5$ & $11.0 \pm 2.5$ & NS \\
\hline Serum E2 level on the day of hCG $(\mathrm{pg} / \mathrm{mL})$ & $\begin{array}{c}3678.2 \\
(185.0-15000.0)\end{array}$ & $\begin{array}{c}4893.2 \\
(526.0-16899.0)\end{array}$ & $<0.01$ \\
\hline $\begin{array}{l}\text { No. of oocytes retrieved } \\
\text { Fertilization method }\end{array}$ & $9.7 \pm 6.7$ & $12.5 \pm 6.5$ & $<0.01$ \\
\hline $\operatorname{IVF}(\%)$ & $76.4(226 / 296)$ & $78.2(169 / 216)$ & NS \\
\hline ICSI (\%) & $23.6(70 / 296)$ & $21.8(47 / 216)$ & NS \\
\hline Fertilization rate (\%) & $61.2(1760 / 2878)$ & $60.9(1641 / 2696)$ & NS \\
\hline Cleavage rate $(\%)$ & $99.5(1751 / 1760)$ & $99.5(1632 / 1641)$ & NS \\
\hline Day 3 high-quality embryos rate (\%) & $71.0(1243 / 1751)$ & $74.7(1219 / 1632)$ & 0.02 \\
\hline No. of day 3 high-quality embryos & $4.2 \pm 4.2$ & $5.6 \pm 4.1$ & $<0.01$ \\
\hline
\end{tabular}

Table 3: IVF/ICSI-ET outcomes.

\begin{tabular}{lccc}
\hline & Non-OCP group & OCP group & $P$ value \\
\hline No. of initiated cycles & 296 & 216 \\
No. of embryo transfer cycles & 239 & 175 & \\
No. of transferred embryos & $1.6 \pm 0.8$ & $1.6 \pm 0.8$ & $\mathrm{NS}$ \\
Cycle cancelation rate (\%) & $19.3(57 / 296)$ & $19.0(41 / 216)$ & $\mathrm{NS}$ \\
Biochemical pregnancy rate (\%) & $1.7(4 / 239)$ & $5.7(10 / 175)$ & 0.03 \\
Implantation rate (\%) & $32.3(149 / 461)$ & $32.7(112 / 343)$ & $\mathrm{NS}$ \\
Clinical pregnancy rate per initiated cycle (\%) & $37.2(110 / 296)$ & $40.7(88 / 216)$ & $\mathrm{NS}$ \\
Clinical pregnancy rate per embryo transfer cycle (\%) & $46.0(110 / 239)$ & $50.3(88 / 175)$ & $\mathrm{NS}$ \\
Live birth pregnancy rate (\%) & $80.5(120 / 149)$ & $81.3(91 / 112)$ & $\mathrm{NS}$ \\
Miscarriage rate (\%) & $18.8(28 / 149)$ & $16.1(18 / 112)$ & $\mathrm{NS}$ \\
Ectopic pregnancy rate (\%) & $0.7(1 / 149)$ & $2.7(3 / 112)$ & $\mathrm{NS}$ \\
\hline
\end{tabular}

Note: NS = not statistically significant 
effective in endometriosis and can be advantageous for endometriosis because of the shortened time required for follicular maturation and the diminished amount of $\mathrm{rFSH}$ required providing adequate ovarian stimulation. Therefore, GnRH agonist protocol with $\mathrm{OCP}$ pretreatment might be a useful choice of COS for endometriosis. Larger studies with standardized methods will be needed for confirmation of our conclusions.

\section{CONCLUSION}

We demonstrated differences in results of OCP and nonOCP group. Advantages can be compared as; pretreatment with OCP shorter doses and duration of gonadotropin releasing hormone agonist. By using $\mathrm{OCP}$, gonadotropin releasing hormone cycles can be started in a scheduled manner since weekend oocytes retrieval were reduced. This study was limited to retrospective analysis, as a result, there are potential biases. Our patients were identified based on inclusion criteria which is one of the accepted ways of identifying endometriosis who respond poorly. Patients were not randomly assigned to the medications, but it was the choice of the treatment physician since there are no instructional guidelines for the selection of OCP or $\mathrm{GnRH}$ agonist in such patients. Use of OCPs is an acceptable alternative by all most physicians. Cycles with no suppression with GnRH agonist flare use were not included to avoid another factor potentially influencing the outcome. Our results did not show any improvement in cycle outcome and pregnancy rate but definitely showed high quality of oocytes, increased number of oocytes which showed a beneficial effect of OCP in IVF cycle.

\section{REFERENCES}

Bellver, J., Albert, C., Labarta, E., Pellicer, A., (2007). Early pregnancy loss in women stimulated with gonadotropin-releasing hormone antagonis protocols according to oral contraceptive pill pretreatment. Fertil Steril, 87(5), 1098-1101. [DOI]

Bendikson, K., Milki, A.A., Speck-Zulak, A., Westphal, L.M., (2006). Comparison of GnRH antagonist cycles with and without oral contraceptive pretreatment in potential poor prognosis patients. Clin Exp Obstet Gynecol, 33(3), 145-147. PMid:17089576

Biljan, M.M., Buckett, W.M., Dean, N., Phillips, S.J., Tan, S.L., (2000). The outcome of IVF-embryo transfer treatment in patients who develop three follicles or less. Hum Reprod, 15(10), 2140-2144. [DOI]

Davis, L., Kennedy, S.S., Moore, J., Prentice, A., (2007). Oral contraceptives for pain associated with endometriosis. Cochrane Database Syst Rev(3), Cd001019. [DOI]

Dessolle, L., Ferrier, D., Colombel, A., Freour, T., Jean, M., Barriere, P., (2011). Prolonging GnRH-agonist to achieve ovarian suppression does not compromise the results of a long protocol. Eur J Obstet Gynecol Reprod Biol, 159(1), 111-114. [DOI]

Hughes, E., Fedorkow, D., Collins, J., Vandekerckhove, P., (2003). Ovulation suppression for endometriosis. Cochrane Database Syst Rev(3), Cd000155. [DOI]

Lessey, B.A., Young, S.L., (2014). Homeostasis imbalance in the endometrium of women with implantation defects: The role of estrogen and progesterone. Semin Reprod Med, 32(5), 365-375. [DOI]

Levens, E.D., Whitcomb, B.W., Kort, J.D., Materia-Hoover, D., Larsen, F.W., (2009). Microdose follicular flare: A viable alternative for normalresponding patients undergoing in vitro fertilization? Fertil Steril, 91(1), 110-114. [DOI]

Ma, Q.H., Li, S.W., Huang, Z.Y., Han, Z.Y., Qiu, D.S., Han, D.W., (2007). [impact of controlled ovarian hyperstimulation delayed, which occurs after functionally pituitary downregulation by depot GnRH agonist, on the outcome of in vitro fertilization and embryo transfer]. Sichuan $\mathrm{Da}$ Хue Xue Bao Yi Xue Ban, 38(6), 980-983. PMid:18095601

Pluchino, N., Freschi, L., Wenger, J.M., Streuli, I., (2016). Innovations in classical hormonal targets for endometriosis. Expert Rev Clin Pharmacol, 9(2), 317-327. [DOI]

Siristatidis, C.S., Gibreel, A., Basios, G., Maheshwari, A., Bhattacharya, S (2015). Gonadotrophin-releasing hormone agonist protocols for pituitary suppression in assisted reproduction. Cochrane Database Syst $\operatorname{Rev}(11)$, Cd006919. [DOI

Tanbo, T., Fedorcsak, P., (2017). Endometriosis-associated infertility: Aspects of pathophysiological mechanisms and treatment options. Acto Obstet Gynecol Scand, 96(6), 659-667. [DOI]

Venetis, C.A., Kolibianakis, E.M., Tarlatzi, T.B., Tarlatzis, B.C., (2009) Benefits of luteinizing hormone activity in ovarian stimulation for IVF. Reprod Biomed Online, 18 Suppl 2, 31-36. [DOI]

Vercellini, P., Buggio, L., Berlanda, N., Barbara, G., Somigliana, E., Bosari, S., (2016). Estrogen-progestins and progestins for the management of endometriosis. Fertil Steril, 106(7), 1552-1571.e1552. [DOI]

Zegers-Hochschild, F., Adamson, G.D., De Mouzon, J., Ishihara, O., Mansour, R., Nygren, K., Sullivan, E., Vanderpoel, S., (2009). International committee for monitoring assisted reproductive technology (icmart) and the world health organization (who) revised glossary of art terminology, 2009. Fertil Steril, 92(5), 1520-1524. [DOI]

Zhu, X., Ye, H., Fu, Y., (2016). The utrogestan and hmg protocol in patients with polycystic ovarian syndrome undergoing controlled ovarian hyperstimulation during ivf/icsi treatments. Medicine (Baltimore), 95(28), e4193. [DOI] 